\title{
Internet of Intelligences in Risk Analysis for Online Services
}

\author{
Chongfu Huang ${ }^{1,2,3}$ \\ ${ }^{1}$ State Key Laboratory of Earth Surface Processes and Resources Ecology (Beijing Normal University) \\ Beijing 100875, China, hchongfu@bnu.edu.cn \\ ${ }^{2}$ Key Laboratory of Environmental Change and Natural Disaster, Ministry of Education of China, Beijing Normal \\ University, Beijing 100875, China \\ ${ }^{3}$ Academy of Disaster Reduction and Emergency Management, Ministry of Civil Affairs \& Ministry of Education \\ the Peoples' Republic of China, Beijing Normal University, Beijing 100875, China
}

\begin{abstract}
There are always some people who experienced or studied the risks of daily life. Based on that, in this paper, we proposed a new concept "internet of intelligences" (IOI), which consists of intelligent agents, the Internet and a mathematics model. An IOI collects and processes the information provided by the agents with experiences and judgment in a risk. It serves for the customer who hasn't any experience in a specific risk. The key of constructing a positive IOI is to filter malicious interference, process inconsistent information, and carry out an optimal treatment on incomplete information. Its core technology is the data processing method with information diffusion. The online services of IOI can help us, such as choosing health foods, correctly filling in the college candidate voluntary and guaranteeing love affairs more romantic.
\end{abstract}

Keywords: Risk, intelligent agent, Internet, information diffusion, online service.

\section{风险分析在线服务的智联网}

\section{黄崇福 $1,2,3$}

1.北京师范大学地表过程与资源生态国家重点实验室，北京 100875

2. 北京师范大学环境演变与自然灾害教育部重点实验室, 北京 100875

3. 北京师范大学，民政部/教育部减灾与应急管理研究院，北京 100875

摘要：基于总有一些人已经历或研究过日常生活中的风险问题，本文首次提出 “智联网” 的概念，它由 智能体、互联网和一个数学模型构成, 以汇集和处理相关信息, 为面对风险而又没有经验的人们提供服务。过 滤恶意假信息，处理不协调信息，优化处理不完备信息等，是构建正向智联网的关键，其核心技术是信息扩散 的数据处理方法。智联网的在线服务, 有望帮助人们有效解决远离有害食品、正确填报高考志愿和使恋爱更符 合个性等问题。

关键词：风险，智能体，互联网，信息扩散，在线服务

人类社会经历了从农业阶段到工业阶段，再到 信息化阶段三次巨大浪潮。目前, 人类正处于第三 次浪潮之中, 其特点是形形色色的互联网无处不 在。移动通信、物联网、战术数据链等大型的信息 系统, 无一例外皆有互联网的烙印。然而, 今天的 互联网系统, 远非智能系统, 更多的是信息发布和 交换系统。以此支持的在线服务, 并没有超出传统 服务的内涵, 只不过速度更快, 花样更多, 成本更 低。换言之, 如果不计成本和时间, 互联网能提供 的服务, 传统手段均能提供。互联网真正的威力尚 在发掘之中, 智联网或许将成为其突破点之一。

\section{1. 智联网概念}

“智联网” (Internet of Intelligences), 是由各种 智能体, 通过互联网形成的一个巨大网络。其目的 是集小智慧为大智慧, 群策群力, 帮助人们更好地 认识世界, 获得更好的生活质量。

本文首次提出的 “智联网” , 将是继人工智能 和计算智能之后的智能科学大革命, 同时也将推动 世界信息产业再上一个新台阶。智联网的基础是互 联网, 智联网的核心技术是信息扩散的数据处理, 智联网的发展动力是有偿智能服务。 
与传统咨询服务业最大的不同是, 提供智联网 服务的不再限于专家。对某些实际问题, 民众的经 验可能强于专家的知识。对于巨大系统的复杂问 题, 专家群体的意见往往强于个别权威的判断。通 过 “智联网” 获得尽可能正确的明智选择, 将成为 人们日常生活中的重要助手。

设 $A=\left\{a_{1}, a_{2}, \ldots, a_{n}\right\}$ 是含有的 $n$ 个智能体 $a_{1}, a_{2}, \ldots, a_{n}$ (例如某大学的 $n$ 个教授）的一个集合。 设 $N=\left\{S, C_{1}, C_{2}, \ldots, C_{n}\right\}$ 是含有服务器 $S$ 和 $n$ 台计算机网 络终端 $C_{1}, C_{2}, \ldots, C_{n}$ 的, 一个可以独立工作的子系统, 例如一个局域网。设 $M$ 是处理 $n$ 个智能体所提供的 信息的模型。三元体 $(A, N, M)$ 称为一个智联网。

假定存在某一准则 $R$, 可以判定 $a_{i}, i=1,2, \ldots . n$, 的智力水平, 记为 $q_{i}=R\left(a_{i}\right)$ 。假定 $R$ 还能判定三元体 $(A, N, M)$ 的智力水平, 记为 $Q=R(A, N, M)$ 。当 $Q>\max \left\{q_{1}, q_{2}, \ldots, q_{n}\right\}$ 时, 我们称其为一个正向智联 网。

我们可以举例来说明什么是智联网, 什么是正 向智联网。三人团队 $A=\{$ 张三, 李四, 王五 $\}$ 参加过 传统智力竞赛, 即, 现场抢答, 依标准答案现场判 分。接下来, 张三, 李四, 王五改为各使用一台由 服务器 $S$ 管理的网络 $N$ 参赛, 其他参赛队仍沿袭现 场抢答方式。假定我们设计出了某种可以实时处理 $A$ 队所提供信息的模型 $M$, 并依据处理结果回答问 题。由参赛队 $A$, 计算机网络 $N$ 和信息处理模型 $M$ 组成的系统是一个智联网。假定在以往参赛中最高 智力者为张三, 称为 $A$ 队的传统智力分, 记为 $q$ 。 再假定 $A$ 队用网络参赛而得的网络智力分为 $Q$ 。当 $Q>q$ 时, $(A, N, M)$ 就是一个正向智联网, 此时, $A$ 队的得分一定高于传统方式参赛的得分。

最简单的智联网可以用图 1 示之, 该系统由三 个智能体 $a_{1}, a_{2}, a_{3}$, 三台终端机 $c_{1}, c_{2}, c_{3}$, 一台服务器 $S$ 和一个信息处理模型 $M$ 组成。

事实上, 并非智能体联网就能形成智联网。例 如, 医学影像远程网络会诊并不是智联网, 因为系 统中并不存在本质上能对专家意见进行处理的任何 模型。并非智联网均有正向性, 例如, 用一个简单 的统计模型支撑的智联网, 理论上来讲, 智力水平 为网中智能体的平均值。显然, 智联网的核心技术 在于网络信息处理模型 $M$ 。下面我们给出智联网及 其有关概念的形式化定义。

定义 1. 具有观察、演绎、推理和解决问题能力的个 体称为智能体。

例如, 正常的个人、决策支持系统、恒温调节 器、模糊洗衣机等, 均是智能体。

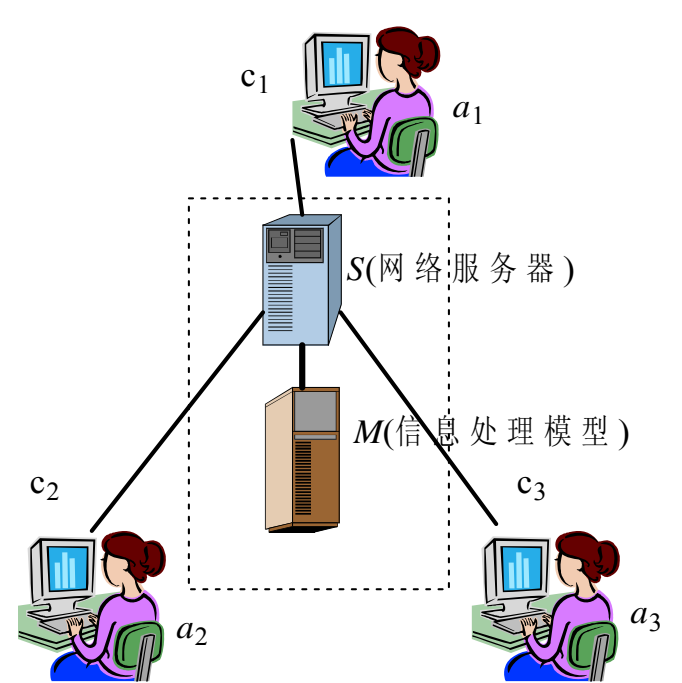

图 1. 最简单的智联网。图中的三台计算机形成网 络, 三个工作人员是三个智能体。

定义 2. 智能体解决问题的能力大小称为智力水平。

例如, 中学生参加智力竞赛的得分高低通常能 反映其智力水平。由于各种智力竞赛的难易程度和 测试方式不同, 由得分反映的智力水平具有相对 性。

定义 3. 设 $A$ 是一个智能体集合, $N$ 是 $A$ 使用的一个 网络, $M$ 是处理 $A$ 所提供信息的模型, 三元体 ( $A$, $N, M$ ）称为一个智联网。

换言之，一个由智能体、计算机网络和信息处 理器组成的系统, 称为一个智联网。当其智力水平 超越每个个体智力水平时, 称为一个正向智联网。 即,

定义 4. 设 $(A, N, M)$ 是一个智联网, 智力水平为 $Q$ 。再设 $A$ 中个体的最大智力水平为 $q$ 。当 $Q$ 大于 $q$ 时, 称 $(A, N, M)$ 为一个正向智联网。

互联网是一个动态混沌系统, 没有人知道每一 个节点何时会发生什么变化, 也不知这种变化对其 它节点会产生什么影响。智联网则是一个智力提升 系统, 只有正向智联网才有存在的意义。智联网具 有动态自组织性, 目标是形成一个高级智能体。互 联网从混沌系统走向自组织系统, 必将是一次革 命。

经过对比互联网应用模式的发展和人类大脑的 结构机理, 人们发现, 二者有惊人的重合。例如, 从电子邮件、电子公告牌到博客、社会化网络, 互 联网的每一个应用创新都能映射到人脑的功能结构 
中 $^{[1]}$ 。人们预测, 互联网的应用模式将形成一个与 人类大脑功能结构高度相似的网络虚拟结构, 互联 网正朝着使人脑充分互联的方向发展和进化。然 而, 现有的互联网技术, 尚不足以自动形成超越个 体智能体的智能, IBM 为其百年大庆于 2011 年推出 的, 能与冠军一比高下的沃森 ${ }^{[2]}$, 不过是以往经验 的学习而已。只有智联网, 才有可能使网络成长为 超级智能体, 也就是个体智能的组合, 超越简单智 能的叠加。

\section{2. 风险分析与智联网}

解决如何识别、规避、权衡风险的问题, 是人 们得以正常生活的基本保障。如果过马路时不能识 别风险, 就可能发生交通事故; 如果地震区的建筑 物不设防, 就无法规避地震风险; 如果感染后过量 吃抗生素, 就会让病菌产生耐药性。解决风险问

题, 基础是风险分析。

使用有关理论和方法, 在相关知识和数据资料 的基础上，对未来不利事件出现的可能性、规模、 影响等进行的分析, 称为风险分析。风险分析是为 了认识风险, 为风险管理提供科学依据, 使未来情 景向好的方向转变。

复杂问题的风险分析, 需要相当的智慧。提高 监测手段、综合使用各种技术和信息、大量访问专 家等, 是目前解决复杂风险问题的主要途径。然 而, 无论监测手段多么完善, 大量的风险分析工作 还是遇到信息不完备的问题; 无论综合多少技术和 信息, 分析中的许多缺陷仍然暴露无遗; 无论访问 多少专家, 结论也不会比简单统计更有说服力。

由于正向智联网能超越个体智力水平, 用智联 网改善现有的风险分析技术, 极具发展前途。相应 地, 我们可以发展出监测型智联网、综合型智联网 和评价型智联网。

\section{1 监测型智联网}

设 $A$ 是含有的 $n$ 个智能监测器（例如一台复杂 机器人配置的 $n$ 个智能传感器) 的一个集合, $N$ 是 连接这 $n$ 个智能监测器的一个计算机网络, $M$ 是处 理 $n$ 个智能监测器所提供的信息的模型, 三元体 ( $A$, $N, M$ ）称为一个监测型智联网。

例如, 当一台机器人识别其环境风险时, 监测 型智联网能提高其识别能力。当我们监测自然和社 会的各个机构能形成监测型智联网时, 我们对自然 灾害风险的监测能力就会大大提高。

监测型智联网正向化的基础是 $M$ 能使监测器联 动起来, 并能生成比单个监测器更具使用价值的信
息。例如, 一个能快速捕捉变化目标的监测器能通 过 $N$ 和 $M$ 使高精度观察器及时联动到目标上来。多 个监测器的信息经 $M$ 处理能对被监测对象有超越直 观监测的认识。

监测型智联网有可能推进物联网的智能化。地 震监测智联网有可能为提高地震预报精度创造条 件。

\section{2 综合型智联网}

设 $A$ 由 $n$ 个智能体组成，他们分别使用不同的 技术 (例如, 线性回归技术、人工神经元网络技术 等) 或信息源(例如, 遥感信息、气象信息等) 对某 一风险问题进行研究, $N$ 是连接这 $n$ 个智能体的一 个计算机网络, $M$ 是综合处理它们所提供信息的模 型, 三元体 $(A, N, M)$ 称为一个综合型智联网。

例如, 某灾害管理机构建有 “天一地一现场” 一体化业务平台, 分析人员分别使用无人飞机、卫 星遥感和地面观测数据等对气象灾害风险进行分析 并通过计算网络传送给综合模型 $M$ 实时处理, 这就 形成一个综合型智联网。

从分布式处理、并行处理和网格计算发展而来 的云计算, 将在综合型智联网方面发挥重要作用, 因为系统的综合度越高, 实时处理的任务越重。

\section{3 评价型智联网}

设 $A$ 由 $n$ 个评价人员组成, $N$ 是连接他们的一 个计算机网络, $M$ 是处理 $A$ 所提供评价结果的模 型, 三元体 $(A, N, M)$ 称为一个评价型智联网。

例如, 根据欧盟预防和控制工业风险的塞维索 指令 II, 八位专家分别对某化工厂进行风险评价, 并通过计算网络传送给模型 $M$ 进行处理, 这就形成 一个评价型智联网。

与传统的综合处理专家评价结果最大的不同 是, 评价型智联网不仅使专家间的匿名提示、协 调、互动更加方便、有效, 也为评价结果超越参与 专家的水平创造了条件。

\section{3. 由智联网支撑的风险评价在线服务}

风险是与某种不利事件有关的一种未来情景 [3]。由个人或社会观察或感觉到的某种事件称为情 景, 它必须由包含时间、地点、对象等要素的系统 来加以描述。“有关”之程度应该用某个度量空间来 测度，例如，概率。“不利事件”的强度应该被量 化, 例如, 损失的量。显然, 任何已发生的灾难都不 是风险; 未来的不同时段, 风险也不同。 
显然, 风险因人而异。2007 年中石油在国内上 市，随后其股票大跌。对于持有其较多股票的人而 言, 风险很大; 对于不持有其股票的人, 中石油股 票是否大跌，与他们无关，没有风险。

风险问题, 举不胜举, 既有因人而异的风险, 更有大量人们尚无法认识的风险。今天，在似乎人 人都懂风险, 人人都会进行风险评估的时代, 五花 八门的预测模型、拟合模型、插值模型等被称为是 风险分析模型, 而且, “专家打分” 进行风险评 估, 似乎成了灵丹妙药。然而, 现实情况是, 人们 对风险的研究尚处于初级阶段, 这不仅反映在人类 甚至于没有一个共同认知的风险概念, 而且对地震 风险、经济风险等重大风险问题也缺少令人信服的 分析理论和方法, 每每在灾难来临前侥幸多于掌 控。

不过, 在大量风险问题中, 简单风险在一定的 条件下可以被正确认识并加以规避。简单风险是指 人们有能力认识的风险。条件是曾经有人经历过, 并想出了规避的方法。有经验积累的简单风险, 对 经历过的人而言, 能造成伤害的可能性大大降低, 而对没有经历过的人，风险就比较大。

例如, 摸黑走过一条左边有大坑的路, 曾经掉 进坑里的人知道走左边有危险, 应该绕着走, 但是 对从来没有走过的人，他摸黑走该路就有较大风 险, 他或许掉进坑里去, 或许顺利通过。这里, 危 险是指确定性的伤害, 风险是指与伤害有关的一种 未来情景。由于前进路线的不确定性, 伤害可能出 现, 也可能不出现。

简单风险, 也可能涉及复杂的系统, 以个人的 经验, 尚难全面认识。特别地, 当风险系统随着时 间发生明显变化时, 个人经验可能失效。此时, 用 评价型智联网集成风险经历者的经验, 并尽量对集 成经验加以提升, 就能提供风险评价的在线服务。 有三类简单风险较适合于在线服务：信心类简单风 险、决策类简单风险和浪漫类简单风险。

\section{1 信心类简单风险在线服务}

信心的最精辟定义出自《圣经- 希伯来书》: “信心是所望之事的实底, 是未见之事的确据。” 换言之, 信心就是人所希望实现的事情的根据, 是 还没有发生、没有看见的事情必然要发生和被看见 的证据。

信心的来源和本义是宗教领域根本性的问题。 德国威丁堡大学的马丁・ 路德教授在 1517 年的宗教 改革运动中提出的 “因信称义” 的学说认为, 人单 单凭着信仰, 无需教会这个媒介, 就能够直接与上 帝相沟通。只要有信心，而无需其他的条件和资
格, 就能获得生命信仰上的解脱, 获得上天对你的 拯救。于是, 信心成为衡量一个人信仰的标准, 惟 有信心能成就一个人的信仰，而其他所有外在的行 为、条件、资格、外力都不能成为信仰的依据。信 心也成为人在世间生活、工作是否有意义的根据, 成为能够战胜艰难险阻、开拓出美好喜乐的生活的 动力。信心在人类史上开始具有了神圣的意味, 成 为人们生活和工作当中的强大精神力量。今天的清 教徒, 是最为虔诚的新教徒, 他们完全秉持 “因信 称义” 的学说, 他们在信仰方面非常强调信心的作 用。正是凭着这种信心，清教徒们不远万里到北美 拓荒。他们坚信, 这里肯定会是一个繁荣兴旺、日 新月异的新国度; 正是这种信心，使他们获得了独 立战争的胜利, 创建了美利坚合众国。可以说, 是 信心成就了清教徒, 是信心成就了美利坚。

然而, 世俗生活中的信心并不等同于宗教上的 信心。毫无根据的盲信, 足以带来重大伤害。在日 常生活中, 我们对一些事有信心, 因为我们有较充 足的证据信任它; 对一些事则没有信心，感觉到有 些把握不住, 接触它可能会造成伤害。例如, 我们 对中国乒乓球队信心十足，因他们在世界乒乓球锦 标赛上连年夺冠。但是, 我们对中国的食品安全整 体没有信心, 因为转型期的中国对食品安全的监管 严重缺失。2008 年爆发的三聚氨胺污染事件极大地 打击了人们对国产奶粉的信心，全国有近 30 万名婴 幼儿因食用问题奶粉患泌尿系统结石 ${ }^{[4]}$ 。2011 年曝 光的 “全国每年返回餐桌的地沟油 200 万到 300 万 吨 ${ }^{[5]}$ ”似乎说明中国的食品安全几近失控。

在宗教上, 人的信心像芥菜一样, 开始看起来 比较小, 但未来成就非常巨大, 超过开始时几百甚 至几万倍, 人就应该拥有这种必将成就万千成果的 信心。信心的大小不是按目前的想法比较的, 而是 按照将来要成就的成果来比较大小的。人虽然比山 渺小, 但他如果有移山的信心, 他的信心就比巍峨 的大山还要大。

在世俗生活中, 人的信心像凭据一样, 信心的 大小是由目前的处境决定的。信心由三个因素决 定: 行动实现难度的认知、个人或团体的情绪和外 在意识。认知是指人们对行为必定成功的认识过 程, 由于这种认识过程只能是对行为未来发展状况 的预期, 所以这种认识过程实际上又是一种对行为 过程的想象和推断; 情绪是指有机体在受到生活环 境中的刺激时, 生物需要是否获得满足而产生的暂 时的较剧烈的评价和体验, 它包括喜、怒、忧、 思、悲、恐、惊七种; 外在意识是指人们在行为中 大脑对外界事物觉察的清醒程度和反应灵敏程度, 
人们在睡眠时意识水平最低, 在注意力高度凝聚时 意识水平最高。

与风险有关的信心问题，其实就信心的逆问

题。以人们对消费品的信心为例, 如果有大量的真 凭实据表明某一消费品存在明显的安全问题, 消费 者使用它就面对较大的被伤害风险。这时人们对此 消费品的信心就低; 反之, 如果风险很小, 信心就 高。

信心类简单风险在线服务, 就是构建一个计算 信心指数的信息处理模型 $M$, 由其支撑一个智联网 $(A, N, M)$ 。

信心指数原本是债券市场上的一个指标, 定义 为 10 个顶级公司债券的平均收益率与 10 个中等级 别公司债券的平均收益率的比值。这个比值总是小 于 $100 \%$, 因为高级别债券所承诺提供的到期收益率 较小。当债券交易者对经济表示乐观时, 他们对低 级别债券所要求违约溢酬率就较低, 这两类债券的 收益率差就变小, 信心指数接近 $100 \%$ 。因此, 较高 的信心指数通常是牛市的信号。这里的模型 $M$ 可表 为

\section{信心 $=\frac{10 \text { 个顶级公司债券的平均收益率 }}{10 \text { 个中等公司债券的平均收益率 }}$}

如果从风险分析的角度来看待消费品的信心指 数, 我们可将其简单地定义为消费满意度。当所有 消费者都 $100 \%$ 地满意时, 信心指数为 1 ; 当所有消 费者都绝对不满意时, 信心指数为 0 。

现实中的问题是, 任何调查机构都只能采取抽 样方式进行调查, 并且大多数机构的委托人是商品 生产者, 调查机构为了迎合雇主的需要, 无法确保 调查结果的客观性。另一个更为棘手的问题是, 调 查数据中常常夹杂有许多不实信息, 如何排除, 并 非易事。我们可以用智联网技术很好地解决这些问 题。

设 $O$ 是待评估对象, $A$ 是由 $n$ 个消费者组成的 智能体, 他们经由互联网分别根据自己的消费经历 对 $O$ 进行定性和定量描述及评价。由于智联网的开 放性和成本低, 调查的面可以很广。当我们使用基 于信息扩散 ${ }^{[6]}$ 技术对调查进行分析后, 就可以得知 消费群体想表达的意思, 从而评估出他们对 $O$ 的信 心。设 $O_{1}, O_{2}, \ldots, O_{t}$ 是由智联网评估的 $t$ 个同类消费 品, 将它们依信心指数的大小从上到下排序, 就能 提供风险在线服务。

经由智联网提供这类服务的重点是个性化。人 们从信心指数排名上只能看到综合而笼统的结果, 并不提供性价比参数, 更没消费品适合对象的信 息。两个信心指数相近的消费品, 可能价格相差较
大。信息回溯功能不仅可以让潜在消费者根据自己 选定的指标进行评价, 还可以看到一些事故案例。 当自己能承受时, 信心指数随即提高。

作者建议首先建立奶粉信心智联网和饮料信心 智联网, 并可以尝试建立城市级餐馆信心智联网。

支撑信心类简单风险在线服务的智联网，是一 种评价型智联网, 其中的智能体是普通的消费者, 使用的是互联网, 评价模型 $M$ 和信息回溯功能决定 服务质量。

\section{2 决策类简单风险在线服务}

决策, 是指管理主体为了实现某种目标而对未 来一定时期内有关活动的原则、方法、技术、途径 等拟定备选方案, 并从各种备选方案中作出选择的 活动。备选方案是指供选择用的行动措施 (原则、 方法、技术、途径等）的汇集。原则上，决策可通 过下述四步来实现。

第一步：目标拟定。根据管理主体需要解决的 问题, 提出管理目标。例如, 防灾减灾部门依据国 家的法律、法规，并根据可用资源、基本组织架构 等, 确定工作方针、减灾目标、长远减灾规划等, 从而提出监测、预警、应急的目标等等。灾后 24 小 时内救灾物资到达灾民手中，就是民政部门应急管 理的一个具体目标。

第二步：资料分析。根据管理目标，通过各种 途径和渠道, 收集管理系统内部的和外部的数据资 料和相关信息。并进行必要的整理和分析。显然, 收集到的资料和信息越多、越准确，通过分析对自 然状态的认识和未来情景的预测也就越接近客观实 际，所作出的管理决策也就越合理。所以，在管理 决策过程中，资料和信息收集是十分重要的。但需 要特别注意的是, 由于时间紧迫和成本等问题, 资 料和信息收集难以做到又多又准，应适可而止。当 然, 资料和信息的收集, 一方面要有目的、有针对 性地进行收集、整理、分析; 另一方面, 也要依靠 平时的积累和存储。

第三步: 寻求备选。所谓制定备选方案, 就是 以所要解决的问题为目标, 对收集到的情报和信息 资料认真整理、分析和科学计算, 并以此为依据制 定出几个实现目标的方案, 提交管理决策者选定。 制定备选方案, 也是一项比较复杂, 要求较高的重 要工作, 有时还需采用试验的方法, 有的要采用数 学的方法, 进行可靠性和可行性分析, 提出每个方 案的利与弊, 然后才能提供备选。例如, 自然灾害 风险评价模型的选用, 就是一个典型的寻求备选问 题。面对大千世界, 风险评价模型举不胜举。所要 解决的问题不同，所能使用的数据资料不同，可供 
选择的风险模型种类也不同。如果是为保险公司计 算标的费率所用, 概率类模型应为备选。

第四步：最优选项。这里所说的选定最优方

案, 是在若干个备选方案中, 选定一个最佳方案。 这是管理决策的最后阶段, 也是关键的一环。管理 决策的成功与否, 直接关系着管理涉及的企业或社 会系统的发展, 关系到职工或民众的切身利益, 甚 至往往决定着企业或社会的命运。

传统上, 上述的决策工作都是管理主体组织专 家来进行。由于管理系统的复杂性, 人们越来越难 以选出最优方案, 更多的时候是选出满意方案。人 们在这方面做了很多有益的探索。其中, 遗传算法 就能为寻找满意方案提供许许多多帮助。

这种途径只适于重大项目的决策, 最大问题是 高成本。然而, 人们在现实生活中常常遇到一些个 人的决策问题, 不当的决策潜伏着巨大的风险。另 一方面, 大量的人或许已经历类似的决策, 或对其 有了相当的研究, 可以用智联网来形成重要的经 验。

设 $P$ 是某人待决策的问题, $A$ 由 $n$ 个经历和思 考过此问题的人组成, 他们经由互联网 $N$ 分别对 $P$ 进行阐述, 并根据自己的经历或思考提出对 $P$ 的决 策建议。由于智联网可以提供足够大的信息兼容空 间, 参与者可以用智联网平台提供的标准构件, 规 范但较自由地表述对 $P$ 的决策建议。智联网上的标 准构件和处理它们的算法, 构成了模型 $M$ 。当 ( $A$, $N, M$ ）为一个正向智联网时, 它能给需要决策的人 提供重要帮助。

“志愿无忧网” (http://www.51bzy.com/), 以专 家咨询的方式为千千万万的高考学子提供填报志愿 的咨询, 很受考生家长们的欢迎。但是, 这类网上 咨询服务, 与传统咨询服务并无本质区别, 只是当 事人见面的方式有所不同。这种网上咨询, 无法提 高产品质量, 但有利于扩大市场。

用智联网辅助决策, 能集思广义, 可望大幅提 高产品质量, 极可能将专家咨询提升到一个高度。 当我们使用基于信息扩散的核心技术对参与者提供 的信息进行分析后, 就可以得知不同备选方案对面 的风险, 从而给使用者提供不同条件下采取何种决 策方案的建议。更进一步地, 可以根据模糊风险分 析原理, 列出其它参考方案。

作者建议首先建立高考志愿智联网和专业取向 智联网, 并可以尝试为年青学者建立选择科研方向 的智联网。

支撑决策类简单风险在线服务的智联网，是一 种综合型智联网, 其中的智能体是有一定背景的智
者, 使用的是互联网。如何过滤误导信息, 融合同 类有效信息, 是模型 $M$ 必须考虑的重大问题。

\section{3 浪漫类简单风险在线服务}

风险渗透于生活的方方面面。谈情说爱、旅游 观光等浪漫的事, 时常也不尽如人意, 一些严重的 后果甚至会使人痛苦一生。有效规避相关的风险, 锦上添花才能实现。

设 $L$ 是某人拟全程经历的浪漫之旅, $A$ 由 $n$ 个 经历过浪漫之旅或有相当研究的人组成, 他们经由 互联网 $N$, 比对 $L$ 与自己的经历或重要案例, 从而 判断参与者是否适合此浪漫之旅, 并提出建议。由 于智联网能溶百家之精华, 显旅程之弯曲, 无疑可 以帮助浪漫中人提前看清旅程。如果前面是大坑, 停止前进; 如果略有阻力, 则设法解决。由于浪漫 之旅的动态性, 这类风险问题的在线服务, 可能要 有一定的持续性。智联网 $(A, N, M)$ 中的信息处理 模型 $M$, 将更具挑战性。

我们以高校学生恋爱为例来说明模型 $M$ 的基本 原理。首先是 $L$ 的构建。它由两大部分组成: 双方 自然属性和社会发展属性。前者由家庭背景（居住 地、社会地位和经济条件等) 和个人特质 (学习成 绩、性格和身体条件等）构成; 后者由社会价值取 向的变化和双方今后可能的就业构成。接下来是经 历过浪漫之旅或有相当研究的人能剖析 $L$ 和进行对 比并给出建议的空间 $x$ 。设 $x_{1}, x_{2}, \ldots, x_{n}$ 是由 $n$ 个智 能体在 $t$ 时给出的信息, $F\left(t, L, x_{1}, x_{2}, \ldots, x_{n}\right)$ 是对其处 理的结果。由于两人相互了解决的加深, 在下一个 时刻, $L$ 会有所变化, 一些信息会得到补充, 一些 信息会得到进行更正, 形成新的 $L^{\prime}$ 。此时, 当持 $L^{\prime}$ 再次上智联网时, 假定另有 $n$ '个智能体在 $t^{\prime}$ 时给出 信息 $x_{1}^{\prime}, x_{2}^{\prime}, \cdots, x_{n^{\prime}}^{\prime}$, 则我们有 $F\left(t^{\prime}, L^{\prime}, x_{1}^{\prime}, x_{2}^{\prime}, \cdots, x_{n^{\prime}}^{\prime}\right)$, 模型 $M$ 就是要处理一系列这样的结果, 给当事人提 供非常负责的建议。

作者建议首先建立高校学生恋爱智联网和 “新 加坡、马来西亚、泰国” 旅游智联网, 并可以尝试 建立国际旅游智联网。

支撑浪漫类简单风险在线服务的智联网, 是一 种监测型智联网, 其中的智能体是有过浪漫经历或 相当研究的人, 使用的是互联网。根据形势变化提 供较为稳定的服务, 是模型 $M$ 必须考虑的问题。

\section{4. 智联网中的关键技术}

古人云: 三个臭皮匠，顶一个诸葛亮。古人又 云：一个和尚挑水吃, 两个和尚抬水吃, 三个和尚 
没水吃。前者是说, 集思广义能出大智慧; 后者是 说, 如果没有好的管理, 人多了会坏事。

显然, 使智联网呈正向, 即, 智联网 ( $A, N$, $M ）$ 的智力水平大于其中任何一个智能体 $a_{i}$ 的智力 水平, 是智联网技术能投入实用的关键。今天, 智 能体遍布大江南北, 长城内外, 世界各地; 另一方 面, 让智能体 $a_{i}$ 上网已经没有任何技术问题。也就 是说, 智联网 $(A, N, M)$ 中的 $A$ 和 $N$ 已经没有什么 问题, 关键在于 $M$ 。

传统的信息处理模型, 大多假定其处理的数据 信息是基本可靠的, 协调性较好的, 关注的是信息 中的模式, 例如各种函数关系。

智联网中的 $M$ 则完全不同，面对的可能是恶意 假信息, 还有客观存在的不协调性, 其次才是有效 信息的处理, 并且信息永远不完备。因此, 智联网 中的关键技术，其实就是解决上述问题的技术。

\section{恶意假信息识别技术}

设 $x_{i}$ 是来自于智联网 $(A, N, M)$ 中智能体 $a_{i}$ 的 信息, 如何判定它是恶意假信息? 最简单的技术是 离差技术。设样本 $X=\left\{x_{1}, x_{2}, \ldots, x_{N}\right\}$ 的中心点是 $x_{0}$, 如果 $x_{i}$ 与 $x_{0}$ 的距离超过其它点与 $x_{0}$ 平均距离一定倍 数, 则判定它是恶意假信息。

关键的问题还在于样本点的全面量化以及缺失项 的处理, 涉及不完备信息, 需用到信息扩散技术。

\section{不协调信息识别技术}

如果 $X=\left\{x_{1}, x_{2}, \ldots, x_{n}\right\}$ 中有大量的不协调信息, 则是智能体间不同看法的正常反映。用模型自动找 出相对协调的子集, 并形成新的样本供更高一个层 次的模型学习, 是解决此问题的较好策略。扩散型 人工神经元网络 ${ }^{[7]}$ 已经提供了这方面的技术。

\section{不完备信息处理技术}

当 $X=\left\{x_{1}, x_{2}, \ldots, x_{n}\right\}$ 是可以直接使用的信息时, 由于智联网成本的控制, $n$ 是一个较有限的整数, 相应的信息 $X$ 源是不完备的。无论是用其估计概率 分布还是识别因素间的关系, 均是小样本问题, 须 用信息扩散技术处理。

以信息扩散原理为基础, 人们已经建立了五个 实用的分析模型:

(1) 用信息分配方法计算软直方图, 比传统直方图 的估计效率提高 $28 \%$;

(2) 自学习离散回归模型, 可以直接从给定的样本 生成表达输入输出关系的模糊关系矩阵;

（3）基于正态扩散函数的一种混合型人工神经元网 络, 有效地解决矛盾样本的学习问题;
（4）以历史灾情资料为依据的农业自然灾害风险评 估模型, 计算以县市为区域的自然灾害风险水平;

（5）计算模糊风险的内集一外集模型，不仅可以替 代专家依据给出的样本进行模糊概率估计, 而且实 践证明, 用这一模型计算出来的自然灾害模糊风险 能对减灾方案进行合理的篮选。

上述模型经过一定的改造就可用于智联网，只 不过是将自然灾害的数据改为来自智联网的数据。 尤其是自学习离散回归模型, 非常适合于无人操作 的系统大量处理数据。

\section{5. 人类将进入智联网时代}

1712 年托马斯 - 纽科门制造了早期的蒸汽机。 从 1765 年到 1790 年, 詹姆斯 - 瓦特运用科学理 论, 使蒸汽机的效率提高到原来纽科门机的 3 倍 多, 最终发明出了现代意义上的蒸汽机。1 807 年罗 伯特 - 富尔顿第一个成功地用蒸汽机来驱动轮船。 由于蒸气机的出现, 人类脱离了繁重的体力劳动, 进入了机械化时代, 也称为蒸汽机时代或第一次工 业革命。

从19 世纪 60-70 年代开始, 出现了一系列电气 发明。1866 年德国工程师西门子制成发电机, 1870 年比利时工程师格拉姆发明电动机, 电力开始用于 带动机器, 成为补充和取代蒸汽动力的新能源。电 力工业和电器制造业迅速发展起来, 人类跨入了电 气化时代。电力的广泛应用、内燃机的发明和新交 通工具的发明应用, 称为第二次工业革命。

1946 年由冯・诺依曼设计的世界上第一台电子管 电子计算机在美国诞生, 其快速运算的能力解决了 过去人力所不能完成的大量计算工作。从此, 人类 进入计算机时代。晶体管电子计算机的出现, 使计 算机开始在工业、农业、商业、医学、军事、科研 等领域发挥巨大。微处理芯片的出现, 使计算机深 入到人们的家庭和日常生活。

1974 年美国国防部国防前沿研究项目署

（ARPA）的罗伯特 - 卡恩和斯坦福大学的温顿 - 瑟 夫开发了 TCP/IP 协议, 定义了在电脑网络之间传送 信息的方法, ARPA 网在内部投入使用。1990 年欧 洲核子研究组织的蒂姆 - 伯纳斯-李推出世界上第一 个网页浏览器和第一个网页服务器, 推动了万维网 的产生, 人类进入互联网时代。尽管 1995 年至 2000 年间大量投机的出现产生了网络经济泡沫, 最 终导致 NASDAQ 和所有网络公司崩溃, 但互联网的 发展速度并没有减慢。今天, 便捷的互联网不仅催 生了电子商务、网络文化等诸多产业, 而且产生了 许多像 MySpace、Facebook、Youtube、维基百科这 
样的虚拟社区。随着互联网用户的增加, 互联网在 现代经济生活中正发挥着日益重要的作用。截至 2010 年 6 月 30 日，中国的网民达 4.2 亿人，互联网 普及率达到 $31.8 \%$, 继续超过世界平均水平 ${ }^{[8]}$ 。

今天的互联网, 仍以承载信息流为主, 尚不具 备本质上的智能。然而, 一旦终端上的智能体之智 慧能借助互联网联接起来并被放大, 智联网时代就 将来临。如果说 TCP/IP 协议使互联网成为可能, 则 解决终端智能协议远不能成就智联网, 更关键的是 处理相关信息的模型 $M$ 。不同类型的智能问题, 相 应的模型应该不同。不远的将来, 云计算和物联网 中的模型也有智能化的可能, 将它们溶入智联网, 将加速智联网时代的到来。届时, 人类个体有限的 智慧有可能被溶合后产生高级智慧, 甚至大量出现 不亚于爱因斯坦的超级智慧。果真如此的话, 人类 的生活会更加美好, 人类的未来会更加光明。

\section{6. 结论与讨论}

将智能体用计算机联接起来的网络称为智联 网。智联网将是继人工智能和计算智能之后的智能 科学大革命。

智联网的发展动力是有偿智能服务。与传统咨 询服务业最大的不同是, 提供智联网服务的不再限 于专家，而主要是大量有经验的民众。

智联网 $(A, N, M)$ 正向否, 取决于处理 $n$ 个智 能体所提供信息的模型 $M$ 。对于提供风险评价在线 服务的智联网，信息扩散理论和方法成为 $M$ 的核心 技术，以解决信息不完备和自学习的问题。

本文首次提出了智联网的概念, 并针对风险分 析在线服务展开了研究, 但仍处于理论探索阶段, 尚未建成一个运行的智联网。作者建议以最简单、 最明了的情景展开实验, 形成简单的 $M$ 范式。

一个最简单的实验如下：实验主持人邀请 $A, B$, $C, D$ 四人参加实验, 选定一条正在施工的道路作为 实验对象。

主持人让 $A$ 白天对施工道路的路况进行详细考 查, 并写出报告备用。主持人让 $B, C, D$ 三人夜晚通 过施工的道路, 最先通过者给予奖励。随后, 主持 人让这三人描述道路路况, 描述得最详细的给予奖 励。并不知道路况的主持人综合 $B, C, D$ 三人提供的 信息, 形成报告。最后, 主持人研究 $A$ 提供的报告 和自己综合的报告, 从而解决以下两大问题: 1 . 让 $B, C, D$ 写什么形成的报告才更于自己综合出较完整 的报告; 2 . 仅仅根据三人提供的信息, 如何让自己 的综合报告与 $A$ 提供的报告差异最小。
显然, 第一个问题的解决, 有利于设计智联网 终端的交互界面; 第二个问题的解决, 则有利于形 成 $M$ 的内核。

人工智能经历了从形式化到专家系统再到计算 智能的漫长发展过程, 效果并不显著, 目前仍是步 履维艰。无论知识工程也好, 人工神经元网络也 罢, 不仅无法生产出超过普通人的实用智能系统, 大多数系统甚至连少儿的智能都不如。智联网的出 现, 有望推动人工智能获得长足发展。

风险分析是展示人类智慧最具挑战性的问题之 一, 智联网集思广义的能力, 有望使人们在日常生 活中从未知的风险世界进入已经的风险世界，从而 在别人的帮助下可以自由地选择是去担当风险还是 规避风险。担当风险的背后可能有较大收益, 规避 风险则降低不必要的伤害。

\section{参考文献}

[1] 刘锋, 彭柫, 刘颖. 从人脑的结构机理看互联网的进化. 人类工效学, 2009, 15(1): 11-14.

[2] 苏锋. “沃森” 的胜利. 微电脑世界, 2011,(3): 2 .

[3] Huang C. F. and Ruan D. Fuzzy risks and an updating algorithm with new observations. Risk Analysis, 2008, 28(3): 681-694

[4] 赵新培. 问题奶粉致婴儿泌尿系统结石, 全国发现 29.4 万名. 北京青年报, 2008 年 12 月 2 日.

[5] 汪挺. 食用油市场利润遭受严重挤压, 正规军染指地沟 油生产源于暴利. 中国商报, 2011 年 9 月 20 日.

[6] Huang C. F. and Shi Y. Towards Efficient Fuzzy Information Processing --- Using the Principle of Information Diffusion. Heidelberg: Physica-Verlag (Springer), 2002.

[7] Huang, C.F. and Leung, Y. Estimating the relationship between isoseismal area and earthquake magnitude by hybrid fuzzy-neural-network method. Fuzzy Sets and Systems, 1999, 107(2): 131-146.

[8] 祝华新, 单学刚, 胡江春. 2010 年中国互联网舆情分析 报告. 人民网, [2011-01-23] http://www.people.com.cn/ GB/209043/210110/13740882.html 